\title{
Testosteron macht Sildenafil nicht wirksamer
}

\section{Zur Korrektur der erektilen Dysfunk- tion ist bei Männern mit niedrigeren Testosteronspiegeln die Kombina- tion aus Sildenafil und Testosteron nicht erfolgreicher als eine Sildenafil- Monotherapie.}

- In einer randomisierten, doppelblind geführten und placebokontrollierten Studie wurden 140 Männer zwischen 40 und 70 Jahren mit einem Score von 25 oder weniger auf der erektilen Dysfunktionsskala und Testosteronwerten unter $11,45 \mathrm{nmol} / \mathrm{l}(330 \mathrm{ng} / \mathrm{dl}) 14$ Wochen lang entweder mit einem täglich applizierten testosteronhaltigen Gel oder Placebo behandelt. Alle Männer lebten in einer stabilen Partnerschaft.

Die Ausgangs-Dysfunktionsscores beider Gruppen waren zu Beginn der Studie gleich. Wie zu erwarten ging die Anwendung von Sildenafil in der Monotherapie mit einer signifikanten Verringerung des Dysfunktionsscores auf im Mittel 7,7 einher. Die Änderung dieses Scores erfolgte aber in beiden
Gruppen in gleicher Weise unabhängig davon, ob die Männer zusätzlich noch das Testosterongel oder ein Placebogel anwandten.

Obwohl die Serum-Testosteronkonzentrationen unter der Substitution um im Mittel 22,5 nmol/l (649 ng/dl) anstiegen, hatte Testosteron nicht nur keinen Effekt auf die erektile Dysfunktion, sondern leistete im Gegensatz zur landläufigen Meinung auch keinen nennenswerten Beitrag zur Libido, zur allgemeinen Sexualfunktion, zur Häufigkeit und Befriedigung sexueller Begegnungen und zur allgemeinen Vitalität. Interessant ist der Umstand, dass es bereits in der drei bis sieben Wochen dauernden offenen Sildenafil-Vorlaufphase zu einem signifikanten Anstieg der Testosteronwerte um 3,47 nmol/l (100 ng/dl) kam. Über diesen Effekt lässt sich trefflich spekulieren. Möglicherweise regen die neu gewonnenen Fähigkeiten zum Vollzug auch die Keimdrüsen zu erhöhter Aktivität an. Wer gefordert wird, der liefert auch mehr.

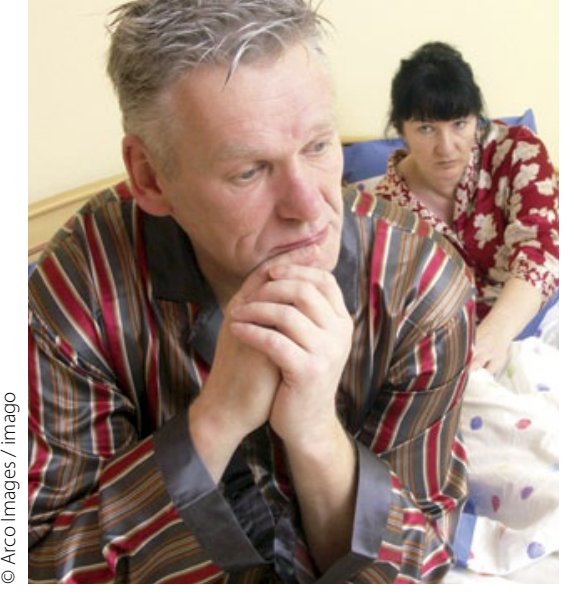

Leistet Testosteron gar keinen nennenswerten Beitrag zur Libido?

- M. Spitzer et al.

(Korres.: matthew.spitzer@bmc.org): Efect of testosterone replacement on response to sidenafil citrate in men with erectile dysfunction. Ann. Int. Med. 2012; 157: 681-691

\section{Kommentar}

Natürlich ist die Studie kein Argument gegen die Testosteronsubstitution bei Männern mit deutlich erniedrigten Spiegeln. Sie ist aber sehr wohl ein Argument gegen die routinemäßige Anwendung von Testosteron zur Verbesserung der erektilen Dysfunktion bei Männern mit nur leicht oder gar nicht erniedrigten Testosteronwerten. Zusätzlich liefert sie Belege dafür, dass sexuelle Aktivität und befriedigendes Sexualleben mit den Testosteronkonzentrationen doch nur in einem sehr lockeren Zusammenhang stehen!

H. S. FÜESSL "

\section{Hilft Homöopathie bei Chemotherapie?}

\begin{abstract}
Kann ein homöopathisches Komplexmittel, das vor allem gegen Reisekrankheit eingesetzt wird, Krebspatienten helfen, die wegen der Chemotherapie unter Übelkeit leiden?
\end{abstract}

- Französische Onkologen haben 431 Brustkrebspatientinnen, die mit Chemotherapie behandelt wurden, in zwei Gruppen randomisiert. Die Experimentalgruppe erhielt während der Behandlung ein homöopathisches Komplexmittel, das in Frankreich gegen Übelkeit und Erbrechen zugelassen ist. Die Kontrollgruppe nahm während des gleichen Zeitraums Placebos ein. Alle Patienten wurden zusätzlich lege artis behandelt.

Hauptzielparameter dieser Studie war die Übelkeit, die mit einem vali- dierten Score ermittelt wurde. Die Auswertungen zeigen, dass die homöopathische Therapie dem Placebo nicht überlegen war - auch dann nicht, wenn in einer Subgruppenanalyse nur die besonders stark betroffenen Patienten in Betracht gezogen wurden.

\begin{abstract}
- D. Pérol et al.
Can treatment with Cocculine improve the control of chemotherapy-induced emesis in early breast cancer patients? A randomized, multi-centered, double-blind, placebo-controlled Phase III trial. BMC Cancer. 2012 Dec 17; 12: 603. doi: 10.1186/1471-2407-12-60
\end{abstract}

\section{Kommentar}

Das hier getestete homöopathische Komplexmittel, Cocculine, enthält Cocculus indicus $4 \mathrm{CH}$, Strychnos nux vomica $4 \mathrm{CH}$, Nicotiana tabacum $4 \mathrm{CH}$, Petroleum rectificatum 4CH aa 0,375 mg. Einige Homöopathen werden daher monieren, dass eine solche Therapie nicht den Regeln der klassischen Homöopathie entspricht; daher, so könnten sie argumentieren, spricht das vorliegende negative Ergebnis nicht gegen die Wirksamkeit der Homöopathie. Ich würde dem entgegnen, dass in diesem Fall die Homöopathie sich eindeutig gegen solche Komplexmittel aussprechen sollte. Solange sie das nicht tut, muss diese sehr gut gemachte Studie als ein weiterer Beweis für die Unwirksamkeit der Homöopathie gelten.

E. ERNST 